\title{
Electron Beam Effects on Kinetic Alfven Waves in the Plasma Sheet Boundary Layer
}

\author{
G. Shrivastava, J. Shrivastava* \\ ${ }^{1}$ Department of Physics, Vikram University, Ujjain, India \\ ${ }^{2}$ Department of Physics, Dronacharya Group of Institutions, Greater Noida, India \\ Email: ${ }^{*}$ jayashrivastava2004@yahoo.co.in
}

Received 5 July 2014; revised 2 September 2014; accepted 5 October 2014

Copyright (C) 2014 by authors and OALib.

This work is licensed under the Creative Commons Attribution International License (CC BY). http://creativecommons.org/licenses/by/4.0/

\section{(c) (i) Open Access}

\begin{abstract}
This work studies the effect of earthward electron beam on kinetic Alfven wave in plasma sheet boundary layer (PSBL). The particle aspect approach is adopted to investigate the trajectories of charged particles in the electromagnetic field of kinetic Alfven wave. Expressions are found for the dispersion relation, growth/damping-rate and associated currents in the presence of electron beam in homogenous plasma. Kinetic effects of electrons and ions are included to study kinetic Alfven wave because both are important in the transition region. The plasma parameters appropriate to plasma sheet boundary layer are used. It is found that downward electron beam affects the dispersion relation, growth/damping-rate and associated currents in both cases (warm and cold electron limit).
\end{abstract}

Keywords

Kinetic Alfven Wave, Electron Beam, Plasma Sheet Boundary Layer

Subject Areas: Plasma, Theoretical Physics

\section{Introduction}

Plasma sheet boundary layer (PSBL) is located at altitudes between the auroral acceleration region at distances of 1.5 - 3 RE and the more distant portions of the geomagnetic tail where energy conversion processes associated with reconnection occur. Plasma in the geomagnetic is dynamic and high-speed ion flow is frequently detected near the boundary of plasma sheet and the lobe [1]-[3]. The plasma exhibits complex distributions with anisotropies and beams [4] [5]. Both earthward and tailward travelling beams and flows have been observed. The plasma sometimes includes $\mathrm{He}^{++}$and $\mathrm{O}^{+}$ions [6] [7]. Takada et al. [8] recently reported that the cold ion energi-

${ }^{*}$ Corresponding author.

How to cite this paper: Shrivastava, G. and Shrivastava, J. (2014) Electron Beam Effects on Kinetic Alfven Waves in the Plasma Sheet Boundary Layer. Open Access Library Journal, 1: e518. http://dx.doi.org/10.4236/oalib.1100518 
zation occurs simultaneously with large amplitude low-frequency electromagnetic waves accompanied by an intense collimated ion beam.

Kinetic Alfven waves are of great importance in laboratory and space plasmas. These waves may play an important role in energy transport, in driving field-aligned currents, in particle acceleration and heating, and in explaining inverted-V structures in magnetosphere-ionosphere coupling and in solar flares and the solar wind [9][11]. Additional support for their role in auroral phenomena comes from global distribution maps at both low (FAST satellite) and (Polar satellite) altitude show that Alfven waves occur on auroral field lines along the entire auroral oval [12]-[15].

Hasegawa (1976) [9] first suggested that small-scale kinetic Alfven waves possessed parallel electric fields and could be an efficient mechanism for accelerating particles on plasma sheet field lines. The kinetic Alfven waves Hasegawa had spatial scales perpendicular to B such that $k_{\perp} \rho_{s}-1$, where $\rho_{s}$ is the ion acoustic gyroradius. This is the "kinetic" limit of kinetic Alfven waves. Subsequent theoretical investigations [10] focused on kinetic Alfven waves in the electron inertial limit $\left(k_{\perp} c / \omega_{p e}-1\right)$ as a mechanism for establishing parallel electric fields capable of accelerating auroral electrons. Considerable experimental evidence has accumulated from the Viking, Freja and FAST spacecraft, as well as low altitude sounding rockets, that kinetic Alfven waves in the inertial limit are an important feature of the altitude range between $1000 \mathrm{~km}$ and $2.5 \mathrm{RE}$. Lysak (1998) [16] and Lysak and Lotko (1996) [17] have presented a model calculation of the properties of kinetic Alfven waves along a magnetic flux tube in which the altitude range encompasses both regimes. More recently Chaston et al. (2003) [18], Chaston (2004) [19] examined the importance of ions and electrons kinetic effects in the acceleration of electrons in small scale Alfven waves above the auroral oval based on FAST satellite observations. Chaston et al. (2003b) [18] combined the electron inertial and electron and ion kinetic corrections to the standard MHD Alfven wave dispersion relation. At high altitudes along auroral field lines in plasma sheet boundary layer, the plasma gradients are relatively weak and a local theory may describe this wave particle interaction [20]. With this assumption in mind, kinetic Alfven waves in the presence of electron beam are examined in the present paper.

In recent past, Alfven waves have been analyzed using particle aspect analysis in view of the auroral acceleration process [21]-[26]. This model is extended in the present paper to address the processes that occur above the auroral acceleration region at altitudes of $4-7 \mathrm{RE}$ at plasma sheet boundary layer. The main advantages of this approach are to consider the energy transfer between wave and particles, along with the discussion of wave dispersion and the growth/damping rate of the wave. The method may be suitable to deal with the PSBL, where particle acceleration is also important along with wave emissions. The results obtained by this approach are the same as those derived using the kinetic approach.

At high altitudes, the plasma parameters vary slowly, and a local approach can be used to investigate the kinetic effects [16] [20]. Thus the purpose of present paper is to apply this particle aspect approach to examine kinetic Alfven waves in the presence of electron beam that propagate along the plasma sheet boundary layer with spatial scale sizes of $\sim 20 \mathrm{~km}$ (close to electron inertial scales and ion acoustic gyroradius) in anisotropic magnetoplasma, using Polar [13]-[15] [27] [28] satellite data. As for heating/acceleration mechanisms of ions in PSBL, the effects of electromagnetic waves still remain to be understood.

In this paper, kinetic effects of electrons and ions are included to study kinetic Alfven wave in the presence of electron beam because both are important in the transition region. The wave propagating obliquely to the ambient magnetic field $\mathrm{B}_{0}$ is considered. The whole plasma has been considered to consist of resonant and non-resonant particles. The resonant particles participate in an energy exchange process, whereas the non-resonant particles support the oscillatory motion of the wave. The organization of the paper is as follows. An introduction is given in Section 1. Section 2 deals with the theory and results and conclusion are presented in Sections 3 and 4.

\section{Theory}

\subsection{Dispersion Relation}

The present work is based upon the particle aspect analysis as adopted by Terashima; Baronia and Tiwari [22]; Shrivastava et al. [24]; Shrivastava and Tiwari [25] [26] and Shrivastava and Shrivastava [29], for the analysis of waves and instabilities. Detailed mathematical analysis of the kinetic Alfven wave, using particle aspect analysis, has been presented by Baronia and Tiwari [22]. By following their approach the full dispersion relation for kinetic Alfven wave in the presence of electron beam in homogeneous plasma with full kinetic effects of ions and electrons by particle aspect approach is obtained as: 


$$
\left[1-\frac{\omega_{i}^{2}}{k_{\Pi}^{2} C_{s}^{2}} \times \frac{\Gamma_{0}\left(\mu_{e}\right)}{\Gamma_{0}\left(\mu_{i}\right)}\{1+\chi Z(\chi)\}\left[1-\frac{\omega \omega_{i}}{k_{\Pi}^{2} V_{A}^{2}} \times \frac{1-\Gamma_{0}\left(\mu_{i}\right)}{\mu_{i}}\right]=\frac{\omega_{i}^{2}}{k_{\Pi}^{2} V_{T \perp i}{ }^{2}} \frac{1-\Gamma_{0}\left(\mu_{i}\right)}{\Gamma_{0}\left(\mu_{i}\right)}\right.
$$

where, $\omega_{i e}=\omega-k_{\Pi} V_{d i e}$ and $V_{d e}=$ Velocity of electron beam

$$
\begin{gathered}
V_{A}^{2}=\frac{c^{2} \Omega_{i}^{2}}{\omega_{p i}^{2}} \text { is the square of Alfven speed. } \\
C_{s}^{2}=\frac{\omega_{p i}^{2}}{\omega_{p e}^{2}} V_{T \Pi e}^{2} \text { is the square of ion-acoustic speed. }
\end{gathered}
$$

$\omega_{p i, e}^{2}=4 \pi N_{0} e^{2} / m_{i, e}, \quad \mu_{i, e}=k_{\perp}^{2} \rho_{i, e}^{2}, \quad \rho_{i}\left(\rho_{e}\right)$ is the thermal ion(electron) gyroradius. $Z(\chi)$ is the plasma dispersion function, $\chi=\omega / k_{\Pi} V_{\Pi e}, V_{\Pi e}=\left(2 T_{\Pi e} / m_{e}\right)^{1 / 2}$ is the electron thermal speed and $\Gamma_{0}(\mu)=e^{-\mu} I_{0}(\mu)$ is the modified Bessel function

If electron gyroradius is small, $\Gamma_{0}\left(\mu_{e}\right) \approx 1$. For cold electron limit ( $\chi \gg 1$ ), the dispersion relation can be

$$
\frac{\omega^{2}}{k_{\Pi}^{2} V_{A}^{2}} \approx \frac{\left(1+k_{\perp}^{2} \rho_{i}^{2}\right)}{\left(\frac{\omega_{i}}{\omega}+\left(\frac{\omega_{e}^{2}}{\omega^{2}}\right) k_{\perp}^{2} \frac{c^{2}}{\omega_{p e}^{2}}\right)}
$$

The dispersion relation of kinetic Alfven wave is similar to that derived by Lysak [16]; Lysak and Song [20] under the approximation, $V_{\text {die }}=0$ using kinetic approach and more accurate that presented by Baronia and Tiwari [22]; Dwivedi et al. [23] using particle aspect analysis. Baronia and Tiwari [22]; Dwivedi et al. [23] have included other wave modes in their dispersion relation thus it is not similar to that derived by Lysak [16]; Lysak and Song [20] using kinetic approach. Furthermore, Baronia and Tiwari [21] and Dwivedi et al. [23] have not presented different calculations for warm and cold electron limit, the dispersion relation is derived for warm electron limit whereas parallel and perpendicular current densities are presented with cold electron limit. The dispersion relation given in "Equation (1)" for kinetic Alfven waves includes full kinetic effects of ions and electrons in homogeneous plasma by particle aspect approach and may be very useful for transition region where both effects are important as observed by Polar/Hydra [30] electric field instrument [31] satellite [13]-[15] [27] [28].

\subsection{Current Density}

To evaluate the perturbed current density per unit wave-length, the following set of equations is used as described by Baronia and Tiwari [22]; Shrivastava and Shrivastava [29]:

$$
\begin{aligned}
& \boldsymbol{J}_{\boldsymbol{i}, \boldsymbol{e}}=\int_{0}^{\lambda} d s \int_{0}^{\infty} 2 \pi \boldsymbol{V}_{\perp} d \boldsymbol{V}_{\perp} \int_{-\infty}^{\infty} d V_{\Pi} e\left[\left(N+n_{1}\right)(\boldsymbol{V}+\boldsymbol{u})-N \boldsymbol{V}\right] \\
& \boldsymbol{J}=\boldsymbol{J}_{\boldsymbol{i}}-\boldsymbol{J}_{\boldsymbol{e}}
\end{aligned}
$$

The current density in z-direction is obtained as:

For warm electron limit $(\chi \ll 1)$,

$$
\boldsymbol{J}_{\mathbf{z}}=-\frac{\lambda}{4 \pi} e \phi_{1}\left[\frac{\omega_{p i}^{2} k_{\Pi}}{m_{i} \omega_{i}}\left\{\frac{k_{\perp}^{2} \rho_{i}^{2}}{V_{T \perp i}^{2}} \varphi_{1}-\frac{k_{\Pi}^{2}}{\omega_{i}^{2}} \phi_{1}\right\}\left(1-k_{\perp}^{2} \rho_{i}^{2}\right)-\frac{\omega_{p e}^{2}}{m_{e}}\left\{\frac{1}{V_{T \perp e}{ }^{2}}\left(\frac{\omega_{e}}{k_{\Pi} V_{T \perp e}^{2}}\right) \varphi_{1}\right\}\right]
$$

For cold electron limit $(\chi \gg 1)$,

$$
\boldsymbol{J}_{\mathbf{Z}}=-\frac{\lambda}{4 \pi} e \phi_{1}\left[\frac{\omega_{p i}^{2} k_{\Pi}}{m_{i} \omega_{i}}\left\{\frac{k_{\perp}^{2} \rho_{i}^{2}}{V_{T \perp i}^{2}} \varphi_{1}-\frac{k_{\Pi}^{2}}{\omega_{i}^{2}} \phi_{1}\right\}\left(1-k_{\perp}^{2} \rho_{i}^{2}\right)-\frac{\omega_{p e}^{2} k_{\Pi}}{m_{e} \omega_{e}}\left\{\frac{k_{\Pi}^{2}}{\omega_{e}^{2}} \phi_{1}-\frac{1}{V_{T \perp e}{ }^{2}} \varphi_{1}\right\}\right]
$$

Similarly for the current density in $\mathrm{x}$-direction

For warm electron limit $(\chi \ll 1)$, 


$$
\boldsymbol{J}_{\boldsymbol{x}}=\frac{\lambda}{8 \pi} e \varphi_{1} \phi_{1}\left[\frac{\omega_{p i}^{2} k_{\perp}}{m_{i} \omega_{i}}\left\{\frac{k_{\Pi}^{2}}{\Omega_{i}^{2}}\right\}\left(1-k_{\perp}^{2} \rho_{i}^{2}\right)-\frac{\omega_{p e}^{2}}{m_{e}}\left\{\frac{k_{\Pi}^{2}}{\Omega_{e}^{2}}\right\}\left(\frac{k_{\perp} \omega_{e}}{k_{\Pi}^{2} V_{T \Pi e}{ }^{2}}\right)\right]
$$

For cold electron limit $(\chi \gg 1)$,

$$
\boldsymbol{J}_{\boldsymbol{X}}=\frac{\lambda}{8 \pi} e \varphi_{1} \phi_{1}\left[\frac{\omega_{p i}^{2} k_{\perp}}{m_{i} \omega_{i}}\left\{\frac{k_{\Pi}^{2}}{\Omega_{i}^{2}}\right\}\left(1-k_{\perp}^{2} \rho_{i}^{2}\right)-\frac{\omega_{p e}^{2}}{m_{e}}\left\{\frac{k_{\Pi}^{2}}{\Omega_{e}^{2}}\right\}\left(\frac{k_{\perp}}{2 \omega_{e}}\right)\right]
$$

Lysak and Song [20] have discussed kinetic approach to Alfven wave that propagate along plasma sheet boundary layer, as observed by Polar/Hydra [30], electric field instrument [31] satellite data [14] [15] [28] but not presented any idea about the associated upward and downward currents as also observed by Polar/Hydra [30], electric field instrument [31] satellite [14] [15] [28]. Perturbed current densities per unit wave-length are obtained here separately for warm $(\chi \ll 1)$ and cold electron limit $(\chi \gg 1)$. Thus current densities per unit wavelength presented in this paper are more accurate as compared to Baronia and Tiwari [22].

\subsection{Energy Balance and Damping-Rate}

The wave energy density per unit wavelength $W_{W}$ is the sum of pure field energy and the changes in energy of the non-resonant particles $W_{i, e}$ [22], which is written as

$$
W_{W}=\frac{\lambda k_{\perp}^{2} \psi_{1}^{2}}{8 \pi}+W_{i, e}
$$

where $W_{i, e}$ is defined by

$$
W_{i, e}=\int_{0}^{\lambda} d s \int_{0}^{\infty} 2 \pi \boldsymbol{V}_{\perp} d \boldsymbol{V}_{\perp} \int_{-\infty}^{\infty} d V_{\Pi} \frac{m_{i, e}}{2}\left[\left(N+n_{1}\right)(\boldsymbol{V}+\boldsymbol{u})^{2}-N \boldsymbol{V}^{2}\right]_{i, e}
$$

The energy associated with the ion and electron components of the non-resonant particles, is given as

$$
W_{i}=\frac{\lambda k_{\Pi}^{2} \psi_{1}^{2}}{16 \pi} \times \frac{\omega_{p i}^{2}}{\omega_{i}^{2}} \Gamma_{0}\left(\mu_{i}\right)
$$

For warm electron limit,

$$
W_{e}=\frac{\lambda k_{\Pi}^{2} \psi_{1}^{2}}{16 \pi k_{\Pi}^{2}} \times \frac{\omega_{p e}^{2}}{T_{\Pi_{e}} / m_{e}}
$$

For cold electron limit,

$$
W_{e}=\frac{\lambda k_{\Pi}^{2} \psi_{1}^{2}}{16 \pi} \times \frac{\omega_{p e}^{2}}{\omega_{e}^{2}}
$$

where, $k=2 \pi / \lambda$. It follows from the above that the major part of the energy is contained in the form of oscillatory motion of non-resonant electrons; hence

$$
W_{W} \approx W_{e} \approx \frac{\lambda k_{\Pi}^{2} \psi_{1}^{2}}{16 \pi} \times \frac{\omega_{p e}^{2}}{k_{\Pi}^{2}\left(T_{\Pi_{e}} / m_{e}\right)}
$$

For the resonant electrons, it is considered that the main contribution arising from the parallel components of the velocities, and expand the integrand around $V_{\Pi}=\omega / k_{\Pi}$ to obtain an expression for energy. Following the procedure as described by Baronia and Tiwari [22]; Dwivedi et al. [23], the energy density per unit wave length of resonant electrons is given as

$$
W_{r \Pi}=\pi \frac{\lambda k_{\Pi}^{2} \psi_{1}^{2}}{8 \pi} \times \omega_{p e}^{2} \times \frac{\omega_{e}^{2} t}{k_{\Pi}^{3} V_{T \Pi e}{ }^{3}} \times \exp \left(-\frac{\omega_{e}^{2}}{k_{\Pi}^{2} V_{T \Pi e}{ }^{2}}\right)
$$

This expression can be utilized for electron acceleration process by kinetic Alfven waves. 
Using the law of energy conservation,

$$
\frac{\mathrm{d}}{\mathrm{d} t}\left(W_{W}+W_{r \Pi}\right)=0
$$

Substituting the expressions for the wave and particle energies, the damping rate (for warm electron limit) is obtained

$$
\gamma=-\pi^{\frac{1}{2}} \frac{\omega_{e}^{2}}{k_{\Pi} V_{T \Pi е}} \exp \left(-\frac{\omega_{e}^{2}}{k_{\Pi}^{2} V_{т \Pi е}^{2}}\right)
$$

In the case of cold electron limit, this relation becomes

$$
\gamma=\pi^{\frac{1}{2}} \frac{\omega_{e}^{4}}{k_{\Pi}^{3} V_{T \Pi e^{3}}} \exp \left(-\frac{\omega_{e}^{2}}{k_{\Pi}^{2} V_{T \Pi e^{2}}^{2}}\right)
$$

For warm electron limit $(\chi \ll 1)$, the results are similar as derived by Baronia and Tiwari [22] under the approximation $V_{d i, e}=0$. Baronia and Tiwari [22] have presented only growth-rate of kinetic Alfven wave in their model whereas the growth/damping-rate of kinetic Alfven wave is obtained in the present paper. In the derivation of the growth/damping-rate in the present paper, it has been assumed that only resonant electrons participate in the energy exchange with the wave, whereas the non-resonant ions and electrons support the oscillatory nature of the wave.

\section{Results and Discussion}

In the numerical evaluation of the dispersion relation, associated currents per unit wavelength, parallel energy of resonant electrons per unit wavelength and growth/damping rate, the following plasma parameters are used suitable for the transient region at 4 - $7 \mathrm{RE}$ in plasma sheet boundary layer [12]-[15] [27] [28].

$$
B_{0}=400 \mathrm{nT} ; c / \omega_{p e}=10 \mathrm{~km} ; k_{\perp} c / \omega_{p e}=4 \mathrm{keV} ; n_{0}=0.3 \mathrm{~cm}^{-3} ; \rho_{i}=20 \mathrm{~km} ; \rho_{e}=0.3 \mathrm{~km} \text {. }
$$

Figure 1 shows the variation of kinetic Alfven wave frequency $\omega$ with $k_{\perp} c / \omega_{p e}$ for different values of earthward directed electron beam velocity $V_{d e}$ at the fixed values of $V_{d i}, T_{i} / T_{e}$ and $\beta$ (in the case of cold electron limit). The dispersion relation given in "Equation (1)" is similar to that derived by Lysak [16]; Lysak and Song [20] by kinetic approach for the warm electron limit under the approximation, $V_{d j e}=0$. Wygant et al. [28] also used this type of dispersion relation for the analysis of Alfven waves at transition region by kinetic approach. It is noticed that the phase velocity of the wave increases with $k_{\perp} c / \omega_{p e}$ and $V_{d e}$ is more effective towards the higher perpendicular wave numbers. Electron beam velocity increases the wave frequency for the fixed values of ion beam

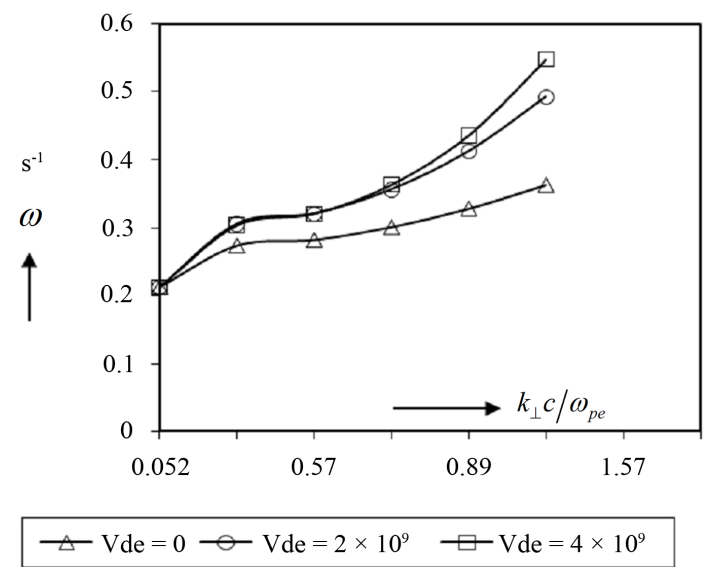

Figure 1. $\omega$ versus $k_{\perp} c / \omega_{p e}$ for different $V_{d e}$ at fixed $\beta=$ 0.001 and the ratio of ion to electron thermal temperature $T_{i} / T_{e}=2, V_{d i}=-1 \times 10^{8} \mathrm{~cm} / \mathrm{sec}$. 
velocity directed towards the magnetotail. Over the last decades it has been established that auroral luminosity is due to the impact of an accelerated electron beam coming towards the earth and at the same events upflowing ion beam has also been observed towards the magnetotail [32] [33] which is considered in present analysis. It is also noticed that waves are low-frequency electromagnetic waves and similar as reported by Takada et al. [34].

The electron beam velocity increases the growth-rate $\gamma / \omega$ as depicted in Figure 2. This may be due to the energy exchange process where only resonant electrons are considered to exchange energy with the wave by inverse Landau damping processes.

Figure 3 presents the increase of $J_{z}$ and Figure 4 presents the increase of $J_{x}$ with the increase of $V_{d e}$ in the presence of $V_{d i}, T_{i} / T_{e}$ and $\beta$ for the cold electron limit. Both the figures predict that the current is generated by kinetic Alfven wave which couples to the perpendicular current. It is noticed that $J_{z}$ is in the upward direction and which is similar as reported by Keiling et al. [15]. It is noticed that the electron beam reduces the upward field-aligned current in a particular range of $k_{\perp} c / \omega_{p e}$ and changes its direction at lower values of $k_{\perp} c / \omega_{p e}$.

\section{Conclusion}

The present study predicts that downgoing electron beam affects the dispersion relation damping/growth-rate

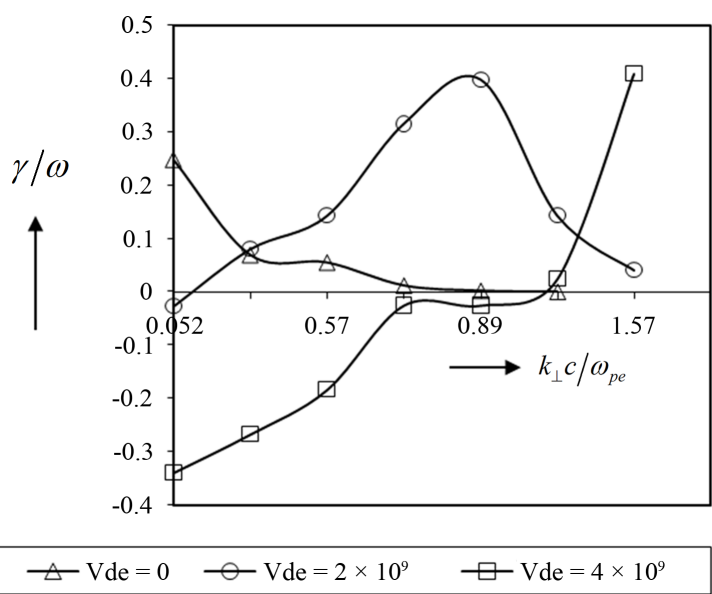

Figure 2. Normalized damping rate versus $k_{\perp} c / \omega_{p e}$ for different $V_{d e}$ at fixed $\beta=0.001$ and the ratio of ion to electron thermal temperature $T_{i} / T_{e}=2, V_{d i}=-1 \times 10^{8} \mathrm{~cm} / \mathrm{sec}$.

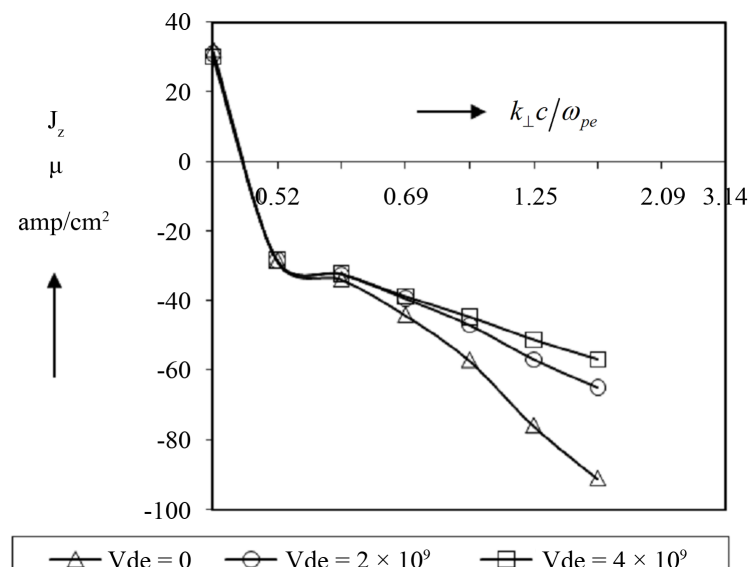

Figure 3. $J_{z}$ versus $k_{\perp} c / \omega_{p e}$ for different $V_{d e}$ at fixed $\beta=$ 0.001 and the ratio of ion to electron thermal temperature $T_{i} / T_{e}=2, V_{d i}=-1 \times 10^{8} \mathrm{~cm} / \mathrm{sec}$. 


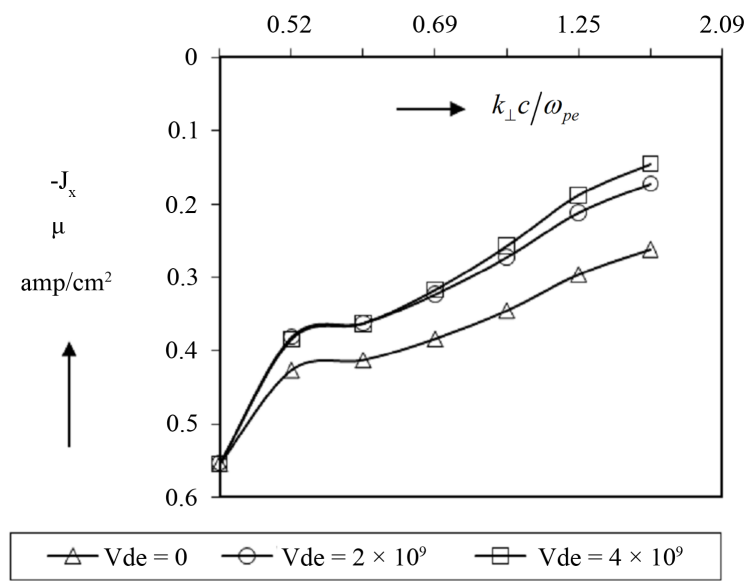

Figure 4. $J_{x}$ versus $k_{\perp} c / \omega_{p e}$ for different $V_{d e}$ at fixed $\beta=$ 0.001 and the ratio of ion to electron thermal temperature $T_{i} / T_{e}=2, V_{d i}=-1 \times 10^{8} \mathrm{~cm} / \mathrm{sec}$.

and associated currents in both cases (warm and cold electron limit). The field-aligned currents, which are the critical features of magnetosphere-ionosphere coupling, may reverse their direction while propagating towards the ionosphere as the magnetic flux tube converges and $\beta$ becomes higher. The present model is based upon the evaluation of charged particle velocity and plasma density in the presence of kinetic Alfven wave in the homogenous plasma which may be useful to estimate particle flux and energy exchange between wave and particle along with the evaluation of dispersion relation, damping/growth rate and associated currents in the presence of electron beam.

\section{Acknowledgements}

Dronacharya group of Institutions, Greater Noida (U.P.), is thankfully acknowledged for providing necessary facility.

\section{References}

[1] Hones, E.W., Asbridge Jr., J., Bame, S., Montgomery, M., Singer, S. and Akasofu, S.I. (1972) Measurements of Magnetotail Plasma Flow Made with VELA4B. Journal of Geophysical Research, 77, 5503-5522. http://dx.doi.org/10.1029/JA077i028p05503

[2] Lui, A.T.Y., Eastman, T., Williams, D. and Frank, L. (1983) Observation of Ion Streaming during Substorms. Journal of Geophysical Research: Space Physics, 88, 7753-7764. http://dx.doi.org/10.1029/JA088iA10p07753

[3] Eastman, T.E., Frank, L.A., Peterson, W.K. and Lennartsson, W. (1984) The Plasma Sheet Boundary Layer. Journal of Geophysical Research: Space Physics, 89, 1553-1572. http://dx.doi.org/10.1029/JA089iA03p01553

[4] Nakamura, M., Paschmann, G., Baumjohann, J. and Sckopke (1991) Ion Distributions and Flows near the Neutral Sheet. Journal of Geophysical Research: Space Physics, 96, 5631.

[5] Takahashi, K. and Hones Jr., E.W. (1988) ISEE 1 and 2 Observations of Ion Distributions at the Plasma Sheet-Tail Lobe Boundary. Journal of Geophysical Research: Space Physics, 93, 8558-8582. http://dx.doi.org/10.1029/JA093iA08p08558

[6] Mobius, E., Ipavich, F., Scholer, M., Gloeckler, G., Hovestadt, D. and Klecker, B. (1980) Observation of a Nonthermal Ion Layer at the Plasma Sheet Boundary during Substorm Recovery. Journal of Geophysical Research: Space Physics, 85, 5143-5148. http://dx.doi.org/10.1029/JA085iA10p05143

[7] Frank, L.A. and Ackerson, K.L. (1971) Observations of Charged Particle Precipitation into the Auroral Zone. Journal of Geophysical Research, 76, 3612-3643. http://dx.doi.org/10.1029/JA076i016p03612

[8] Takada, T., Seki, K., Hirahara, M., Terasawa, T., Hoshino, M. and Mukai, T. (2005) Two Types of PSBL Ion Beam Observed by Geotail: Their Relation to Low Frequency Electromagnetic Waves and Cold Ion Energization. Advances in Space Research, 36, 1883-1889. http://dx.doi.org/10.1016/j.asr.2003.09.075 
[9] Hasegawa, A. and Chen, L. (1975) Kinetic Process of Plasma Heating Due to Alfve'n Wave Excitation. Physical Review Letters, 35, 370. http://dx.doi.org/10.1103/PhysRevLett.35.370

[10] Goertz, C.K. and Boswell, R.W. (1979) Magnetosphere-Ionosphere Coupling. Journal of Geophysical Research: Space Physics, 84, 7239-7246. http://dx.doi.org/10.1029/JA084iA12p07239

[11] Goertz, C.K. (1984) Kinetic Alfvén Waves on Auroral Field Lines. Planetary and Space Science, 32, 1387-1392. http://dx.doi.org/10.1016/0032-0633(84)90081-3

[12] Chaston, C.C. (2004) Reply to "Comment by P. K. Shukla and L. Stenflo on 'Kinetic Effects in the Acceleration of Auroral Electrons in Small Scale Alfvén Waves: A FAST Case Study””. Geophysical Research Letters, 31, 3811. http://dx.doi.org/10.1029/2003GL018974

[13] Keiling, A., Wygant, J.R., Cattell, C., Temerin, M., Mozer, F.S., Kletzing, C.A., Scudder, J., Russell, C.T., Lotko, W. and Streltsov, V. (2000) Large Alfvén Wave Power in the Plasma Sheet Boundary Layer during the Expansion Phase of Substorms. Geophysical Research Letters, 27, 3169-3172. http://dx.doi.org/10.1029/2000GL000127

[14] Keiling, A., Wygant, J.R., Cattell, C.A., Mozer, F.S. and Russell, C.T. (2003) The Global Morphology of Wave Poynting Flux: Powering the Aurora. Science, 299, 383-386. http://dx.doi.org/10.1126/science.1080073

[15] Keiling, A., Parks, G.K., Wygant, J.R., Dombeck, J., Mozer, F.S., Russell, C.T., Streltsov, V. and Lotko, W. (2005) Some Properties of Alfvén Waves: Observations in the Tail Lobes and the Plasma Sheet Boundary Layer. Journal of Geophysical Research: Space Physics, 110, Article No. 10907. http://dx.doi.org/10.1029/2004JA010907

[16] Lysak, R.L. and Carlson, C.W. (1981) The Effect of Microscopic Turbulence on Magnetosphere-Ionosphere Coupling. Geophysical Research Letters, 8, 269-272. http://dx.doi.org/10.1029/GL008i003p00269

[17] Lysak, R.L. and Lotko, W. (1996) On the Kinetic Dispersion Relation for Shear Alfvén Waves. Journal of Geophysical Research: Space Physics, 101, 5085-5094. http://dx.doi.org/10.1029/95JA03712

[18] Chaston, C.C., Bonnell, J., Carlson, C.W., McFadden, J.P., Ergun, R.E. and Strangeway, R.J. (2003) Kinetic Effects in the Acceleration of Electrons by Small Scale Alfvén Waves: A FAST Case Study. Geophysical Research Letters, 30, 1289. http://dx.doi.org/10.1029/2002GL015777

[19] Chaston, C.C., Carlson, C.W., Ergun, R.E., McFadden, J.P. and Strangeway, R.J. (2003) Properties of Small-Scale Alfvén Waves and Accelerated Electrons from FAST. Journal of Geophysical Research: Space Physics, 108, 8003. http://dx.doi.org/10.1029/2002JA009420

[20] Lysak, R.L. and Song, Y. (2003) Kinetic Theory of the Alfvén Wave Acceleration of Auroral Electrons. Journal of Geophysical Research: Space Physics, 108, 8005. http://dx.doi.org/10.1029/2002JA009406

[21] Baronia, A. and Tiwari, M.S. (1999) Kinetic Alfvén Wave in the Presence of Loss-Cone Distribution Function in Inhomogeneous Magnetoplasma-Particle Aspect Analysis. Planetary and Space Science, 47, 1111-1118. http://dx.doi.org/10.1016/S0032-0633(99)00029-X

[22] Baronia, A. and Tiwari, M.S. (2000) Kinetic Alfvén Waves in an Inhomogeneous Anisotropic Magneto Plasma in the Presence of an Inhomogeneous Electric Field: Particle Aspect Analysis. Journal of Plasma Physics, 63, 311-328. http://dx.doi.org/10.1017/S0022377899008272

[23] Dwivedi, A.K., Varma, P. and Tiwari, M.S. (2001) Kinetic Alfvén Wave in the Inhomogeneous Magnetosphere and General Distribution Function. Planetary and Space Science, 49, 993-1003. http://dx.doi.org/10.1016/S0032-0633(01)00008-3

[24] Shrivastava, J., Baronia, A., Varma, P. and Tiwari, M.S. (2001) Effect of Electron Beam and Temperature Anisotropy on Alfvén Wave. Indian Journal of Physics, 75, 117.

[25] Shrivastava, J. and Tiwari, M.S. (2004) Alfvén Wave in Dusty Magnetosphere. Indian Journal of Radio \& Space Physics, 33, 25-31.

[26] Shrivastava, J. and Tiwari, M.S. (2005) Alfvén Wave in the Presence of Parallel Electric Field in the Magnetospheric Plasma. Indian Journal of Radio \& Space Physics, 34, 17-22.

[27] Wygant, J.R., Keiling, A., Cattell, C.A., Lysak, R.L., Temerin, M., Mozer, M.S., Kletzing, C.A., Scudder, J.D., Peterson, W., Russell, C.T., Parks, G., Brittnacher, M., Germany, G. and Spann, J. (2000) Polar Spacecraft Based Comparison of Intense Electric Fields and Poynting Flux near and within the Plasma Sheet-Tail Lobe Boundary to UVI Images: An Energy Source for the Aurora. Journal of Geophysical Research: Space Physics, 105, 18675-18692. http://dx.doi.org/10.1029/1999JA900500

[28] Wygant, J.R., Keiling, A., Cattell, C.A., Lysak, R.L., Temerin, M., Mozer, M.S., Kletzing, C.A., Scudder, J.D., Streltsov, V., Lotko, W. and Russell, C.T. (2002) Evidence for Kinetic Alfvén Waves and Parallel Electron Energization at 5 - 7 RE Altitudes in the Plasma Sheet Boundary Layer. Journal of Geophysical Research: Space Physics, 107, 1201.

[29] Shrivastava, J. and Shrivastava, G. (2008) Kinetic Alfvén Wave in Plasma Sheet Boundary Layer-Particle Aspect Analysis. Planetary and Space Science, 56, 1214-1225. http://dx.doi.org/10.1016/j.pss.2008.04.001 
[30] Scudder, J., et al. (1995) Hydra: A 3-Dimensional Electron and Ion Hot Plasma Instrument for the Polar Spacecraft of the GGS Mission. In: Russell, C.T., Ed., The Global Geospace Mission, Kluwer Academic, Norwell, 495.

[31] Harvey, P., Mojer, F.S., Pankow, D., Wygant, J., Maynard, N.C., Singer, H., Sullivan, W., Anderson, P.B., Pfaff, R., Aggson, T., Pedersen, A., Falthammar, C.G. and Tanskannen, T. (1995) The Electric Field Instrument on the Polar Satellite. Space Science Reviews, 71, 583-596. http://dx.doi.org/10.1007/BF00751342

[32] Parks, G.K., Skoug, R.M., Spencer, S.L., McCarthy, M.P., Larson, D., Lin, R.P., Larson D., McFadden, J., Reme, H. and Sanderson, T.R. (1997) Ion Beams Observed in the near Earth Plasma Sheet Region on May 10, 1996. Geophysical Research Letters, 24, 975-978. http://dx.doi.org/10.1029/97GL00177

[33] Parks, G., Chen, L.J., McCarthy, M., Larson, D., Lin, R.P., Phan, T., Reme, H. and Sanderson, T. (1998) New Observations of Ion Beams in the Plasma Sheet Boundary Layer. Geophysical Research Letters, 25, 3285-3288. http://dx.doi.org/10.1029/98GL02208

[34] Takada, T., Seki, K., Hirahara, M., Fujimoto M., Saito, Y., Hayakawa, H. and Mukai, T. (2005) Statistical Properties of Low-Frequency Waves and Ion Beams in the Plasma Sheet Boundary Layer: Geotail Observations. Journal of Geophysical Research: Space Physics, 110, 2204. http://dx.doi.org/10.1029/2004JA010395 